cacy of a low pressure suction system during its introduction to their gastroenterology day ward.

Methods A low pressure suction controller was plugged in to a standard wall suction point, and adjusted to provide 100 $\mathrm{mm} \mathrm{Hg}$ of suction. Ascites was drained using either a Safe-TCentesis or Bonanno catheter and collected in a Vacsax suction carousel. As per our local protocol, $20 \mathrm{~g}$ of salt poor albumin was given per 31 of ascites drained. Blood pressure and heart rate were recorded every $30 \mathrm{~min}$. The total volume of fluid, duration of drainage and any complications were recorded. Flow rate was then calculated in $1 / \mathrm{min}$. Similar data was also collected from patients undergoing free drainage paracentesis, which was used while all nursing staff were familiarised with the suction.

Results 14 patients ( 9 male, 5 female) with large volume ascites underwent 46 episodes of LVP (29 cases ALD, 11 cases NAFLD, 6 malignant ascites - was albumin given to these malignant patients?).

27 LVPs were performed under suction and 19 under free drainage. Compared to free drainage procedures, suction LVPs took significantly less time (mean duration $233.9 \pm 59.1$ vs $279.7 \pm 61.1 \mathrm{~min}, \mathrm{p}=0.01)$. Suction LVPs drained similar volumes $(10.45 \pm 4.1$ vs $9.6 \pm 3.81$, NS) and achieved similar mean flow rates $(0.048 \pm 0.021$ vs $0.035 \pm 0.0141 / \mathrm{min}, \mathrm{NS})$. One patient undergoing suction LVP developed transient mild abdominal pain that settled spontaneously. No patients in either group experienced significant complications.

Conclusion Low pressure suction paracentesis is significantly faster than free drainage procedures, and can be easily introduced in a day case setting. The benefits to patient and nursing time combined with excellent safety have led the authors to continue low pressure LVP as standard care on their gastroenterology day ward.

Competing interests None.

Keywords large volume paracentesis, low pressure suction.

\title{
PTH-119 INTRODUCTION OF A LOW PRESSURE SUCTION SYSTEM FOR DAY CASE LARGE VOLUME PARACENTESIS: RESULTS FROM A SCOTTISH TEACHING HOSPITAL
}

doi:10.1136/gut.2011.239301.520

E Reed, 1,* A Stanley, ${ }^{1}$ E Forrest, ${ }^{1}$ R Gillespie, 1 M Neilson, ${ }^{1}$ S Barclay ${ }^{1}$ 'Glasgow Royal Infirmary, Glasgow, UK

Introduction In most centres including our own, large volume paracentesis (LVP) has traditionally been performed under free drainage. The authors sought to evaluate the safety and effi- 